\title{
MORSE THEORY IN FIELD THEORY
}

\author{
PETER KOROTEEV AND ANDREY ZAYAKIN
}

\begin{abstract}
We describe correlations functions of topological quantum mechanics (TQM) in terms of Morse theory. We review the basics of topological field theories and discuss geometric and algebraic interpretations of TQM. We prove that correlators in TQM can be expressed via intersection numbers of certain submanifolds of the target space with paths of steepest descent between critical points of a Morse function. In the end we conjecture another correspondence between quantum mechanics correlators and integrals of Massey products of certain cohomology classes.
\end{abstract}

\section{INTRODUCTION}

Topological quantum field theories and topological sting theories originating from the works of E. Witten and others $[9,10,11]$ proved to be extremely helpful in understanding of important mathematical problems.

Main feature of topological field theories (TFT) is independence of correlation functions on metric and coordinates [1]. In TFTs there are no propagating (local) degrees of freedom, vacuum expectation values of operators and transition amplitudes (both further referred to as "correlators") in them are dependent only on topology of the target manifold.

In this paper we employ for our purposes a simple example of TFT - Topological quantum mechanics (TQM) with a BRST-like invariant action. It was shown $[2,5]$ that in zero-dimensional analog of this theory partition function is equal to Euler character of the target manifold.

We have two main aims in this paper: the first is to make manifest the correspondence between TFT and geometry of target manifold; the second aim is to study the correspondence between TFT and a differential graded algebra of cohomology classes on target manifold. The first aim is reached by us within a proof of a reasonable ("physical") level of strictness, whereas the second is only conjectured and studied phenomenologically.

First we propose a geometrical interpretation of TQM developed in $[7,6]$. We prove that there is a correspondence between a special kind observables and 1 codimensional cycles on target manifold. Moreover, transition amplitudes in the theory correspond to intersection indices of paths of steepest descent and cycles. This correspondence is proven using path integral representation of correlation function. Establishing a correspondence between TQM and topology of the target manifold we find a geometrical interpretation of all quantities in the theory. It is also shown that correlator can be introduced independently as an integral of pull-backs of forms corresponding to observables over moduli space of graph embeddings into target manifold. 
In [7] it was shown that correlators in TQM satisfy the so-called anticommutativity equation which is a general property of TQM. This allows us to conclude that the same equation holds for the intersection numbers. Thus an interesting mathematical fact is proven by "physical means".

Second, we formulate a conjecture relating correlators in TQM with algebraic operation - Massey product - on cohomology classes of the target manifold. The conjecture, together with the previous property of correlators, makes it possible to relate Massey products and the intersection numbers.

\section{Topological Quantum Mechanics. Overview}

1.1. The Setup. Quantum Mechanics can be considered as the simplest version of Topological Field Theory (TFT). TQM is based on the following set of axioms

(1) Hamiltonian $H \in \operatorname{End}_{\mathbb{R}}(\mathcal{H})$ acting on a Hilbert space of states $\mathcal{H}=\mathcal{H}_{0} \oplus \mathcal{H}_{1}$ can be represented as

$$
H=[Q, G],
$$

where $Q, G \in \operatorname{End}_{\mathbb{R}}(\mathcal{H})$ are odd nilpotent operators

$$
Q^{2}=0, G^{2}=0,
$$

brackets stand for supercommutator, i.e. for two operators $A, B$ with parities $a, b$ respectively one has

$$
[A, B]=A B-(-1)^{a b} B A
$$

Hamiltonian annihilates vacua space

$$
H \mathcal{H}_{0}=0,
$$

which is postulated to be non-empty. $H$ is positively defined on $\mathcal{H}_{1}$, commutes with parity operator $(-1)^{F}$, whereas $Q$ anticommutes

$$
H(-1)^{F}=(-1)^{F} H, \quad Q(-1)^{F}=-(-1)^{F} Q
$$

Here $F$ is a fermion number.

(2) Observables $\mathcal{O}_{i} \in \operatorname{End}_{\mathbb{R}} \mathcal{H}$ in TQM form the following algebra

$$
\mathcal{O}_{i} \mathcal{O}_{j}=C_{i j}^{k} \mathcal{O}_{k}
$$

where $C_{i j}^{k}$ are its structure constants.

Important property of TQM. In all TFTs correlation functions are independent on coordinates $[1,6]$. But this independence should be treated carefully. In the above setup this property is valid if ${ }^{1}$

$$
\left[Q, \mathcal{O}_{i}\right]=0
$$

then each correlator

$$
\left\langle\mathcal{O}_{i_{1}}\left(t_{1}\right) \ldots \mathcal{O}_{i_{m}}\left(t_{m}\right)\right\rangle=\operatorname{Tr}(-1)^{F} e^{-t_{1} H} \mathcal{O}_{i_{1}} e^{\left(t_{1}-t_{2}\right) H} \ldots e^{\left(t_{m-1}-t_{m}\right) H} \mathcal{O}_{i_{m}} e^{t_{m} H},
$$

\footnotetext{
${ }^{1}$ Operators $\mathcal{O}$ satisfying these equations are referred to as zero-observables.
} 
where trace is taken over the Hilbert space $\mathcal{H}$ is independent on coordinates. But correlator may jump after interchanging of some observables, so their order should be preserved in the consideration.

1.2. Deformation and One-Observables. Let us deform operator $Q$ as

$$
Q \rightarrow Q+\sum T^{A} \mathcal{O}_{A}=Q+\mathcal{O}
$$

where $T_{A}$ are parameters(coupling constants), $\mathcal{O}_{A}$ are zero-observables. Then the Hamiltonian becomes

$$
H=[Q, G] \rightarrow[Q, G]+[\mathcal{O}, G]=H_{0}+H_{1}
$$

Considering $H_{1}$ as interaction Hamiltonian we can rewrite evolution operator and the derivative of 1-point correlator $\mathcal{O}_{1}(t)$

$$
\frac{\partial}{\partial T^{A}}\left\langle\mathcal{O}_{1}\left(t_{1}\right)\right\rangle=-\int_{0}^{t_{1}} d \tau \operatorname{Tr}(-1)^{F} e^{-\left(t_{1}-\tau\right) H}\left[\mathcal{O}_{A}, G\right] e^{-\tau H} \mathcal{O}_{1}=\int_{0}^{t_{1}}\left\langle\mathcal{O}_{A}^{(1)}(\tau) \mathcal{O}_{1}(0)\right\rangle d \tau,
$$

where $\mathcal{O}_{A}^{(1)}(t) \equiv-\left[\mathcal{O}_{A}(t), G\right] d t$ is referred to as 1-observable. One-observable is an 1-form on $\mathbb{R}$.

1.3. Generating Function for Correlators. The following property holds for a correlator[7]

$$
\left\langle\mathcal{O}_{A_{i}}\right\rangle_{B \text { deformed }}^{A}=\frac{\partial}{\partial T^{A_{i}}}\langle\mathcal{O}\rangle_{B \text { deformed }}^{A}=\left\langle\mathcal{O}_{A_{i}} e^{\int_{\mathbb{R}}[\mathcal{O}(t), G] d t}\right\rangle_{B}^{A}
$$

Here $\langle. .\rangle_{\text {deformed }}$ denotes vacuum expectation value in an interacting (deformed) theory, $\langle.$.$\rangle - the same quantity in a free (non-deformed) theory.$

We can expand the exponent in (6) in the following Taylor series according to parameters $T_{A}$

$$
\mathcal{F}_{B}^{A}(T) \equiv\left\langle T\left\{\mathcal{O}_{A_{1}} e^{\int_{\mathbb{R}}[\mathcal{O}(t), G] d t}\right\}\right\rangle_{B}^{A}=\sum_{m=1}^{\infty} \mathcal{F}_{B ; A_{1} \ldots, A_{m}}^{A} T^{A_{2}} \ldots T^{A_{m}},
$$

where the coefficient is expressed via

$$
\begin{aligned}
& \mathcal{F}_{B ; A_{1} \ldots A_{m}}^{A} \equiv \frac{1}{(m-1) !}\left\langle\mathrm{T}\left\{\mathcal{O}_{A_{1}}\left[\prod_{i=2}^{m} \int_{\mathbb{R}}\left[\mathcal{O}_{A_{i}}\left(t_{i}\right), G\right] d t_{i}\right]\right\}\right\rangle_{B}^{A} \\
& =\int_{\mathbb{R}_{+}^{m-1}} d \tau^{1} \ldots d \tau^{m-1}\left\langle\mathcal{O}_{A_{1}} G e^{-\tau_{1} H} \mathcal{O}_{A_{2}}(0) G e^{-\tau_{2} H} \ldots \mathcal{O}_{A_{m}}(0)\right\rangle_{B}^{A}
\end{aligned}
$$

Parameters $T_{A}$ have the meaning of coupling constants here and $T\{.$.$\} stands for chrono-$ logical ordering. The whole expression (7), if interpreted physically, corresponds to the vacuum expectation value of $\mathcal{O}_{A_{1}}$ in the theory with interaction $H_{1}$. If all $\mathcal{O}_{A_{i}}=\mathcal{O}$ are the 
same, the operator $K=\int_{0}^{+\infty} G \exp (-H \tau) d \tau$ being introduced, the above formula can be compactly rewritten as

$$
\mathcal{F}_{B}^{(m) A}=\langle\mathcal{O} K \mathcal{O} \ldots K \mathcal{O}\rangle_{B}^{A},
$$

where $\mathcal{F}^{(m)}$ is a short notation for the value determined in (8).

It was shown in [7] that the following anticommutativity equation holds for correlators

$$
\mathcal{D F}+\frac{1}{2}[\mathcal{F}, \mathcal{F}]=0 \quad \text { or }(\mathcal{D}+\mathcal{F})^{2}=0,
$$

where $\mathcal{D}=C_{A B}^{K} T^{A} T^{B} \frac{\partial}{\partial T^{K}}$ is BRST operator (Chevaller differential). Here $\mathcal{D}^{2}=0$ if $C_{A B}^{K}$ is antisymmetric with respect to $A$ and $B$. (Vacuum indices are omitted here). We shall make use of it later.

\section{Geometrical Interpretation of TQM}

2.1. Path Integral Representation of TQM. In this Section we use a theory which is a particular case of TQM. We are going to proceed in a slightly unconventional way, namely, first defining transition amplitudes and afterwards deriving the action functional from them. This will be done to make the geometrical interpretation of the theory more manifest.

Let $\mathcal{M}$ be a smooth closed oriented Riemannian n-manifold, $f$ is a Morse function on it and $v$ is gradient vector field constructed by means of this Morse function, $\operatorname{CP}(\mathcal{M})$ the space of its critical points ${ }^{2}$. Let $A, B \in \mathrm{CP}(\mathcal{M})$ be critical points with indices $p+1$ and $p, p=0, \ldots, n-1$ respectively, and $\Gamma_{B}^{A}$ be a gradient curve initiating at $A$, terminating at $B$ and satisfying the following set of ODEs

$$
\dot{x}^{i}=v^{i} .
$$

Its solutions are integral curves of $v$, or paths of steepest descent (PSD). The worldsheet of the theory is a line $\mathbb{R}$, targetspace is $\mathcal{M}$ and embeddings $x \in \operatorname{Map}_{B}^{A}$

$$
\left.\operatorname{Map}_{B}^{A}=\left\{x(t) \in C^{\infty}\left(\mathbb{R}^{1}, \mathcal{M}\right)\right), \quad x(-\infty)=A, x(+\infty)=B\right\},
$$

satisfying (11) So we embed a line into $\mathcal{M}$ with fixed images of $\pm \infty$ and $x$ are local coordinates on the targetspace, requiring it to be one of the rigid paths of steepest descent between $A$ and $B$ (fig. 2.1). In further considerations we will imply that the above boundary conditions are satisfied. As for the dimension of the $\operatorname{space}^{M_{a p}} A_{B}$ the following statement is valid[3]

$$
\alpha:=\operatorname{dim} \operatorname{Map}_{B}^{A}=\operatorname{ind} A-\operatorname{ind} B-1 .
$$

For example, for $f$ being height function on manifold $\mathcal{M}=\mathbb{S}^{1} \subset \mathbb{R}^{2}$ we have $\alpha=0$, as it is typical in most cases for $A$ and $B$ with indices different by 1 . There is usually a finite number of paths between them. This explains the term "rigid": these paths of steepest descent cannot be continuously deformed into other paths of steepest descent.

\footnotetext{
$2_{\text {see next subsection for details }}$
} 


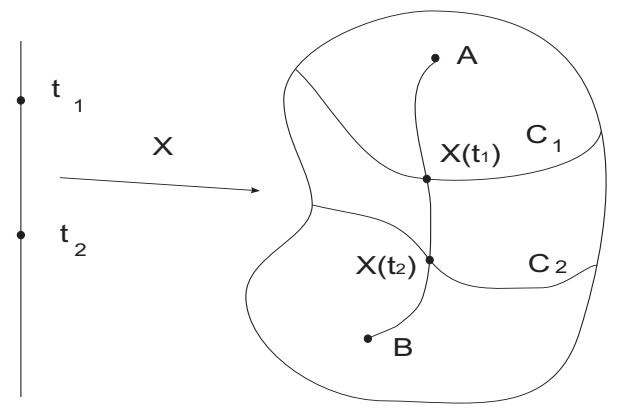

FiguRE 1. Worldsheet and target space of the theory

To develop a quantum theory means to describe the states and transition amplitudes among them. Transition amplitudes are given by path integrals with appropriate boundary conditions. The key point in understanding the geometrical essence of TQM described below is that transition amplitudes in it can be constructed form a purely geometric object. Indeed, consider the following path integral, which does not yet have anything to do with any TFT. At this stage it is just a functional of vector fields on a manifolds $\exp (-S)$, where $S$ is an action is a delta-functional on elements of $\mathrm{Map}_{B}^{A}$ space - space of paths of steepest descent. So we have the following path integral

$$
Z_{A B}=\int \mathcal{D} x \delta[V] \int_{-\infty}^{+\infty} d t \operatorname{det}\left(\nabla_{i} V^{j}(x(t))\right)
$$

where $x(t)$ takes its value in $\mathcal{M}, \nabla_{i}$ is a covariant derivative with Levi-Civita connection and $V=\dot{x}-v$ and $\delta[V]$ is a delta-functional. $V=0$ corresponds to path of steepest descent. The determinant det is a finite-dimensional determinant of matrix $\operatorname{det}\left(\int d t \nabla_{i} V^{j}(x(t))\right)$. The following boundary conditions $x(-\infty)=A, x(+\infty)=B$ are imposed ${ }^{3}$; we remind again that $A, B$ are critical points of the Morse function on the manifold. The measure is here standard Feynman's measure $\mathcal{D} x=\prod_{k=1}^{\infty} d x\left(t_{k}\right)$ with fixed endpoints. By construction this integral counts the number of PSDs with signs. This technique is an infinite dimensional generalization of Mathai-Quillen method[8].

Below it will be made clear that there is a one-to-one correspondence between critical points of Morse function on the manifold and vacua in TQM; moreover, $Z_{A B}$ corresponds to an transition amplitude from vacuum $|A\rangle$ to vacuum $|B\rangle$. The formula (14) can be rewritten as a path integral over even functional variables $\mathcal{D} x \mathcal{D} p$ and odd functional variables $\mathcal{D} \psi \mathcal{D} \pi$, measure $\mathcal{D} x \mathcal{D} \psi$ being measure with fixed endpoints, measure $\mathcal{D} p \mathcal{D} \pi$ being measure with

\footnotetext{
${ }^{3}$ Analogous boundary conditions will be imposed upon each path integral in this paper, unless specified otherwise.
} 
arbitrary endpoints. ${ }^{4}$

$$
Z_{A B}=\int \mathcal{D} x \mathcal{D} \psi \mathcal{D} p \mathcal{D} \pi e^{-S[x(t), p(t), \psi(t), \pi(t)]},
$$

where the functional $S[x(t), p(t), \psi(t), \pi(t)]$ is naturally identified with action and looks like

$$
\begin{gathered}
S=\int_{-\infty}^{+\infty} d t\left[p_{a}\left(\dot{x}^{a}-v^{a}\right)-\psi^{i}\left(\nabla_{i} v^{a}(x)\right) \pi_{a}+\epsilon \psi^{i} \psi^{j} F_{i j}^{b} \pi_{b} \pi_{c} \eta^{a c}+\epsilon p_{a} p_{b} \eta^{a b}\right] \\
=\int p_{a} d x^{a}+\pi_{a} d \psi^{a}-[Q, G] d t=\int P d Q-H d t
\end{gathered}
$$

for generalized super coordinates $Q$ and momenta $P$. $A$ is the affine connection which is related with Levi-Civita connection as $A_{i b}^{a}=e_{k}^{a} e_{b}^{l} \Gamma_{i l}^{k}$ where $g_{i j}=\sum_{a} e_{i}^{a} e_{j}^{a}$. Accordingly, $x(t), p(t), \psi(t), \pi(t)$ are identified with dynamic variables, and $Z$ with transition amplitude, $\epsilon$ plays a role of mass parameter; alternatively, it can be thought of as coupling constant. In (16)

$$
\begin{aligned}
Q & =\psi^{i} \nabla_{i}+p_{a} \frac{\delta}{\delta \pi_{a}}+\psi^{i} \psi^{j} F_{i j b}^{a} \pi_{a} \frac{\delta}{\delta p_{b}}, \\
G & =\pi_{a} v^{a}+\epsilon \pi_{a} p_{b} \eta^{a b},
\end{aligned}
$$

where $F_{i j b}^{a}=\partial_{i} A_{j b}^{a}+A_{i c}^{a} A_{j b}^{c}$ is a curvature tensor.

Critical points $A, B$ are identified with vacua of the theory due to the following reason: in Lagrangian formalism $V(x) \sim\left(v^{i}(x)\right)^{2}$ has the meaning of potential ${ }^{5}$, due to positivedefiniteness, its zeros are its minima; the zeros of a gradient vector field are critical points of the corresponding Morse function.

We provide the following Table of correspondence.

\begin{tabular}{|c|c|c|}
\hline Abstract TQM & PI representation of TQM & Morse theory \\
\hline \hline Vacua space $\mathcal{H}_{0},|A\rangle$ & Minima $A$ of the potential & Critical points CP $(f, \mathcal{M}), A$ \\
\hline Observables $\mathcal{O}$ & $\delta$-functions (1-forms) on cycles & Cycles $C \subset \mathcal{M}$ \\
\hline Amplitude $\langle A \mid B\rangle$ & Amplitude $\langle A \mid B\rangle$ & \# PSDs from A to B \\
\hline Operator $Q$ & $\psi^{i} \nabla_{i}+p_{a} \frac{\delta}{\delta \pi_{a}}+\psi^{i} \psi^{j} F_{i j}{ }^{a} \pi_{a} \frac{\delta}{\delta p_{b}}$ & de-Rham diff. $d$ \\
\hline Operator $G$ & $\pi_{a} v^{a}+\epsilon \pi_{a} p_{b} \eta^{a b}$ & Inner product $\iota_{v}$ \\
\hline
\end{tabular}

2.2. Morse Theory, Witten Complex and $\mathbf{n}$-Matrices. In our theory the space $\mathcal{H}_{0}$ of vacua corresponds to the space of critical points of function $f$ on $\mathcal{M} \operatorname{CP}(f, \mathcal{M})$. So vacua $|A\rangle,|B\rangle$ correspond to critical points $A, B$ and the transition between the initial and the final state corresponds to motion of the point on manifold from $A$ to $B$ by rigid path $\Gamma_{B}^{A}$.

Let $\mathrm{CP}^{i}$ be the linear space of formal linear combinations of all critical points of $\mathcal{M}$ of index $i$. The following complex of chains $\mathrm{CP}^{i}$

$$
\cdots \longrightarrow \mathrm{CP}^{k-1} \longrightarrow \mathrm{CP}^{k} \longrightarrow \mathrm{CP}^{k+1} \longrightarrow \ldots
$$

\footnotetext{
${ }^{4}$ Thus in the formula (15) there is one more integral over $\mathcal{D} p \mathcal{D} \pi$ then over $\mathcal{D} x \mathcal{D} \psi$

${ }^{5}$ This relation becomes apparent after Gauss integration by $p$
} 
is said to be Witten complex $[11,3]$. Here $n\left(n^{2}=0\right)$ is a coboundary operator which increases grading in the complex is given by the explicit formula is

$$
n|B\rangle=\sum_{\Gamma_{B}^{A}} \operatorname{sign} \Gamma_{B}^{A}|A\rangle
$$

where $|B\rangle \in \mathrm{CP}^{i},|A\rangle \in \mathrm{CP}^{i+1}$ and $\operatorname{sign} \Gamma_{B}^{A}$ in the formula (19) gives a sign from each PSD from $A$ to $B$ and it was defined in Witten's paper [11]. If there are several PSDs then their signs are summed up so we can rewrite the formula in the following more convenient matrix notation

$$
n|B\rangle=\sum_{A} n_{B}^{A}|A\rangle,
$$

where $n_{B}^{A}$ is a matrix element, which is equal to the number of PSDs computed with signs, initiating at $A$ and terminating at $B$.

Along with $n_{B}^{A}$ we can introduce $n_{B}^{A}\left(C_{1}, \ldots, C_{m}\right)$ - a number of PSDs from $A$ to $B$ intersecting cycles $C_{1}, \ldots, C_{m} \subset \mathcal{M}$. Here a transversal intersection of 1-dimensional cycles and curve is assumed. Also we consider each cycle intersecting a PSD only once.

Definition 1. Under the above assumptions,

$$
n_{B}^{A}\left(C_{1}, \ldots, C_{m}\right)=\sum_{\Gamma} \prod_{i=1}^{m} \operatorname{ind}\left(C_{i}, \Gamma_{B}^{A}\right),
$$

is said to be higher Morse differential (or n-matrices). Here ind $\left(C_{i}, \Gamma_{B}^{A}\right)$ is an intersection index of the objects into parentheses.

Eventually we have a family of operators, represented by matrices $n_{B}^{A}, n_{B}^{A}\left(C_{1}, \ldots, C_{m}\right)$ $\in \operatorname{Hom}\left(\mathrm{CP}^{i}, \mathrm{CP}^{i+1}\right)$ and as all these objects are nilpotent one can consider a complex for each operator $n_{B}^{A}\left(C_{1}, \ldots, C_{m}\right)$ analogous to Witten complex for $n_{B}^{A}$.

2.3. Correlator via Intersection Numbers. Here we are going to introduce an explicit formula for correlator in Morse theory version of TQM. It will be introduced as a definition but in the next subsection we'll show that the given expression really can be expressed via path integral.

First we take an embedding

$$
x \in \operatorname{Map}_{B}^{A}:=\left\{x \in C^{\infty}(\mathbb{R}, \mathcal{M}) ; x(-\infty)=A, x(+\infty)=B\right\}
$$

for $A$ and $B$ as critical points of certain Morse function and obtain images of points $t_{1}, \ldots, t_{m}$ by this map $x\left(t_{1}\right), \ldots, x\left(t_{m}\right)$. One can treat an evaluation map

$$
\begin{aligned}
e v: \mathbb{R}^{1} \times \operatorname{Map}_{B}^{A} & \longrightarrow \mathcal{M} \\
(x, t) & \longmapsto x(t)
\end{aligned}
$$

We will employ a pull-back of differential forms

$$
e v^{*}: \Omega^{\bullet}(\mathcal{M}) \longrightarrow \Omega^{\bullet}\left(\mathbb{R}^{1} \times \operatorname{Map}_{B}^{A}\right)
$$


For some form $\omega \in \Omega^{\bullet}(\mathcal{M})$ this map is the following ${ }^{6}$

$$
e v^{*} \omega_{I}(x) d x^{I}=\omega_{I}(x(t))\left(\dot{x}^{I}(t) d t+d \varphi^{I}\right),
$$

where differentials $d \varphi^{I}$ belong to $\operatorname{Map}_{B}^{A}$ space.

We construct transversal cycles ${ }^{7}$ to path $\mathrm{AB} C_{1}, \ldots, C_{m}$ on $\mathcal{M}$ so that $x\left(t_{i}\right) \in C_{i}, \operatorname{codim} C_{i}=$ 1. Our next step here is to build closed differential form on $\mathcal{M}$ which is delta-function on a cycle

Definition 2. Form $\omega(x)$ is said to be a delta-form on cycle $C$ and denoted

$$
\omega(x)=\delta_{C}
$$

if

$$
\int_{\mathcal{M}} \omega \wedge \delta_{C}=\int_{C} \omega
$$

And it is a pull-back of delta form which is referred to as observable in TQM.

$$
\mathcal{O}=e v^{*} \omega_{i}
$$

We introduce a new space $\mathfrak{M} \equiv \operatorname{Map}_{B}^{A} \times \mathbb{R}^{1}$. Then the following integral is considered

$$
\int_{\mathfrak{M} \times \mathbb{R}_{+}^{m-1}} e v^{*} \delta_{i_{1}} \wedge \cdots \wedge e v^{*} \delta_{i_{m}} .
$$

Here $\mathbb{R}_{+}^{m-1}$ is a moduli space of embeddings of graphs $t_{1}-t_{2}-\ldots-t_{m}$ into all paths of steepest descent between $A$ and $B$. The substantial statement is that the above integral is equal to $n_{B}^{A}\left(C_{1} \ldots C_{m}\right)$.

Indeed, as Map in our case is zero dimensional and represents a finite set of points and $\mathfrak{M} \times \mathbb{R}_{+}^{m-1}=\operatorname{Map}_{B}^{A} \times \bigsqcup \mathbb{R}_{+}^{m-1} \times \mathbb{R}^{1}$ the integral (26) is equal to the sum of integrals corresponding to each PSD in Map space. Then each term in this sum equals $\prod \operatorname{ind}\left(C_{i}, \Gamma\right)$ by construction. So one has that the integral (26) is equal to $n_{B}^{A}(C \ldots C)$. In fact the integrand after integrating over Map equals wedge product of delta forms on cycles and delta form on PSD. As all cycles are transversal to the path the dimension of the set in the intersection can be equal only to 0 . If there is no intersection of cycle with PSD the answer is 0 for the whole integral and equals 1 if each cycle have an intersection with the curve. In fact the integral under consideration is a sum of \#Map integrals over m-dimensional manifold of m-dimensional delta-function.

\footnotetext{
${ }^{6}$ I don't know how $d \varphi$ can be described rigourously but its particular form won't be important for us 7 thus each cycle has only one common point with AB
} 
2.4. Correlator via Path Integral. Now we will convince the reader that $\mathcal{F}_{B}^{A(m)}$ in (8) is equal to $n_{B}^{A}(\underbrace{C \ldots C}_{m})$ provided an appropriate correspondence for the abstract operator $G \mathcal{O}_{A_{i}}$ is specified in path integral formulation.

Theorem 1. Let $v$ be a smooth vector field on $\mathcal{M}$ and $\mathcal{O}_{A_{i}}^{(1)}$ be 1-observable in TQM. Let $G$ be the inner product $G=\iota_{v}$. Then the following equality between correlator and intersection numbers holds

$$
\mathcal{F}_{B ; A_{1} \ldots A_{m}}^{A}=n_{B}^{A}\left(C_{1}, \ldots, C_{m}\right),
$$

Proof. One can see that the following correspondence arises from the theorem

$$
G \mathcal{O}_{A_{i}}=\delta\left(x^{n}(0)-x^{n}\left(t_{i}\right)\right) v^{n}\left(x\left(t_{i}\right)\right)
$$

Here local coordinates are chosen in such a way that vector field $v$ on $\mathcal{M}$ in the vicinities of intersection points with path of steepest descent has the only one nonzero component $v^{n}, x^{n}$ is the n-th component of coordinate $x$, and $x\left(t_{i}(\tau)\right)$ are images of points $t_{1}, \ldots, t_{m}$ dependent on $\tau$ by embedding $x .^{8}$ One can see that after applying inner product $\iota_{v}$ to one observable a terrible differential $d \varphi$ vanishes.

Representation of the correlator (8) via path integral yields

$$
\mathcal{F}^{(m)}=\int_{\mathbb{R}_{+}^{m-1}} d \tau_{2} \ldots d \tau_{m} \int e^{-S} \mathcal{O}_{A_{1}} \mathcal{O}_{A_{2}}^{(1)} \ldots \mathcal{O}_{A_{m}}^{(1)} .
$$

Here $\tau_{i}=\left|t_{i}-t_{i-1}\right|, i=\overline{2, m}$ parameterize the moduli space of embeddings of $\mathbb{R}$ with marked points $t_{1}, \ldots, t_{m}$ into target manifold $\mathcal{M}$ (see Subsections 2.2 and 2.3).

Then the correlator expansion coefficient can be expressed via path integral

$\mathcal{F}_{B}^{(m) A}=\int d \tau_{2} \cdots d \tau_{m} \int \mathcal{D} x \mathcal{D} p \mathcal{D} \psi \mathcal{D} \pi \exp \left[-\int_{-\infty}^{\infty} d t\left(p_{a}\left(v^{a}(x)-\dot{x}^{a}\right)+\pi_{a} \dot{\psi}^{a}-\psi^{i}\left(\nabla_{i} v^{a}\right) \pi_{a}\right)\right]$

$$
\times \delta\left(x^{n}(0)-x^{n}\left(t_{1}(\tau)\right)\right) \prod_{i=2}^{m} \delta\left(x^{n}(0)-x^{n}\left(t_{i}(\tau)\right)\right) v^{i}\left(x^{n}\left(t_{i}(\tau)\right)\right),
$$

As the operators contain no dependence on Grassman fields $\psi(t), \pi(t)$, one can integrate them out, resulting in $\operatorname{det}\left(\partial_{\tau} \delta_{i}^{j}-\partial_{i} v^{j}\right)$ in the numerator. The integral over $p(t)$ can also easily be done, simply by the definition of delta-functional. Therefore,

$$
\begin{aligned}
\mathcal{F}_{B}^{(m) A} & =\int d \tau_{2} \cdots d \tau_{m} \int \mathcal{D} x \operatorname{det}\left(\partial_{t} \delta_{i}^{j}-\partial_{i} v^{j}\right) \delta[\dot{x}(t)-v(x)] \\
\times & \delta\left(x^{n}(0)-x^{n}\left(t_{1}(\tau)\right)\right) \prod_{i=2}^{m} \delta\left(x^{n}(0)-x^{n}\left(t_{i}(\tau)\right)\right) v^{n}\left(x\left(t_{i}(\tau)\right)\right) .
\end{aligned}
$$

\footnotetext{
${ }^{8}$ by $\tau$ we imply $\tau_{2}, \ldots, \tau_{m}$
} 
One can take the integral over $\mathcal{D} x$ away by virtue of the delta-functional, replacing $x(t)$ by $\mathrm{x}(t)$ - solution of classical Lagrange-Euler equations (11). However, special cares should be taken due to the presence of zero modes in these solutions. Hence, an integral over the space of collective coordinates $\lambda$ remains after integrating the infinite-dimensional $\mathcal{D} x$ integral [1]. Geometrically $\lambda$ corresponds to shift of all points $t_{1}, \ldots, t_{m}$ along $\mathbb{R}$ keeping distances between each other constant and parameterizes second multiplier in the definition of $\mathfrak{M}$ (see 2.3). Reexpressing the delta-functional

$$
\delta\left[\dot{x}^{i}(0)-v^{i}(x)\right]=\sum_{\Gamma} \frac{\delta\left[x^{i}(0)-\mathrm{x}(t)\right]}{\left|\operatorname{det}\left(\partial_{t} \delta_{k}^{j}-\partial_{k} v^{j}\right)\right|},
$$

one cancels determinants (as it should be in a supersymmetric theory) up to sign $(-)^{a}=$ $\operatorname{sign}\left(\operatorname{det}\left(\partial_{t} \delta_{k}^{j}-\partial_{k} v^{j}\right)\right)$ and obtains

$$
\begin{gathered}
\mathcal{F}_{B}^{(m) A}=\sum_{\Gamma} \int d \tau_{2} \cdots d \tau_{m} \int d \lambda(-)^{a} \delta\left(x_{\Gamma}^{(c l) n}(0)-\mathrm{x}\left(t_{1}(\tau), \lambda\right)\right) \\
\prod_{i=2}^{m} \delta\left(\mathrm{x}(0)-\mathrm{x}\left(t_{i}(\tau), \lambda\right)\right) v^{n}\left(\mathrm{x}\left(t_{i}(\tau)\right)\right)
\end{gathered}
$$

Integral over $\lambda$ plays a crucial role here. It allows us to integrate out all the delta-functions, so that a regular expression remains. The latter integral possesses structure absolutely similar to that of the integral (26), which was obtained within a purely geometric construction of section (2.3). Indeed, sum over $\Gamma$ and the integral over zero mode $\lambda$ are equivalent to integration over $\mathfrak{M}$, whereas the integrals over $\tau_{i}$ are taken over same manifolds $\mathbb{R}_{+}^{m-1}$. One can make sure that the following integral

$$
\int d \tau_{i}(-)^{a} v^{n}\left(\mathrm{x}\left(t_{i}(\tau)\right)\right) \delta\left(\mathrm{x}(0)-\mathrm{x}\left(t_{i}(\tau)\right)\right)=\operatorname{ind}\left(\Gamma, C_{i}\right)
$$

is an intersection index between $\Gamma$ and $C_{i}$. Therefore, one comes using (33) to the following expression

$$
\mathcal{F}_{B}^{(m) A}=\sum_{\Gamma} \prod_{i=1}^{m} \operatorname{ind}\left(\Gamma, C_{i}\right)=n_{B}^{A}(C \ldots C) .
$$

Trivially generalizing this result, we thus have proven that for an arbitrary number of cycles,

$$
\mathcal{F}_{B ; A_{1} \ldots A_{m}}^{A}=n_{B}^{A}\left(C_{1}, \ldots, C_{m}\right)
$$

2.5. Generating function for $\mathbf{N}$-matrices. The correspondence described in the above subsection is very useful and has interesting consequences. Indeed as $\mathcal{F}^{(m)}=n_{B}^{A}\left(C_{1} \ldots C_{m}\right)$ one can rewrite for intersection numbers all relations valid for the correlators as well. First 
we mean the anticommutativity equation. As before we construct a generating function namely the whole matrix of them

$$
\begin{aligned}
N_{B}^{A}(T) & :=n_{B}^{A}+n_{B}^{A}\left(C_{i}\right) T^{i}+n_{B}^{A}\left(C_{j}, C_{k}\right) T^{j} T^{k}+\ldots \\
& +n_{B}^{A}\left(C_{p}, \ldots, C_{q}\right) T^{p} \ldots T^{q}+\cdots=\sum_{k} n_{B}^{A}\left(C^{(k)}\right) T^{(k)}
\end{aligned}
$$

where $t$ is a parameter. Actually several nonequivalent cycles are admitted so one needs introducing the same number of parameters. The above construction is an element of the space $\operatorname{Mat}_{N \times N} \otimes \mathbb{R}\left[T^{1} \ldots T^{l}\right]$. Here $l$ is a number of nonequivalent cycles on the manifold.

As anticommutativity equation holds for $N$ (D is taken from (10))

$$
[\mathcal{D}+N, \mathcal{D}+N]=0 \quad \text { or } \quad \mathcal{D} N+\frac{1}{2}[N, N]=0
$$

we obtain interesting relations on intersection numbers in every order in $T$. These equations are indeed very interesting relations in the intersection theory.

\section{Algebraic interpretation of TQM}

Massey product is defined as follows

Definition 3. Let $\alpha \in H^{p}(\mathcal{M}), \beta \in H^{q}(\mathcal{M}), \gamma \in H^{r}(\mathcal{M})$ and $\alpha \beta=0, \beta \gamma=0$. Then Massey product $M P(\alpha, \beta, \gamma)$ is an element of the following quotient space

$$
H^{p+q+r-1}(\mathcal{M}) /\left[\alpha \smile H^{q+r-1}(\mathcal{M})+H^{p+q-1}(\mathcal{M}) \smile \gamma\right]
$$

Let cocycles $a, b, c$ be representatives of $\alpha, \beta, \gamma$ and cochains $u, v$ such that $d u=a b$ and $d v=b c$. Then the cochain $-u c+(-1)^{p}$ av is a cocycle and its cohomological class represents $M P(\alpha, \beta, \gamma)$.

Higher Massey products are defined inductively via products of less order Massey products. However, for higher order Massey products to exist it is necessary for the lesser order Massey products to be trivial. Massey product enables us to determine homotopic class of the manifold up to torsion group.

One can see that the above construction is not well defined. Nevertheless, this problem can be solved introducing the so-called modified Massey product. We need $\alpha \beta, \beta \gamma$ vanished in cohomologies. If they are nonzero the above definition fails. So we introduce the following operator

$$
K=d^{-1} \circ\left(i d-\operatorname{Pr}_{H}\right)
$$

where $\operatorname{Pr}_{H}$ is a projection operator on de-Rham cohomologies ${ }^{9}$. So for each form $\omega$ one has $\left(i d-\operatorname{Pr}_{H}\right) \omega=d \chi$ being exact, the operator $d^{-1}$ is well defined and $K \omega=\chi$. But another problem arises here. Form $\chi$ is not closed any more.

\footnotetext{
${ }^{9}$ Cohomologies here are treated as vector space which
} 
3.1. Conjecture. As there is an embedding of the space of critical points of the Morse function $\mathrm{CP}^{\bullet} \hookrightarrow H^{\bullet}(\mathcal{N})$ in de Rham cohomology groups of $\mathcal{M}$ (as liner spaces) one can make the following

Conjecture 1. Let $A \in C P^{p+1}$ and $B \in C P^{p}$ be critical points of indices $p+1$ and $p$ respectively and $|A\rangle \in \mathcal{H}_{0}^{p+1},|B\rangle \in \mathcal{H}_{0}^{p}$ be representatives of vacua space.

There is a correspondence between observables in TQM and forms in de Rham cohomology groups of $\mathcal{M}$

$$
\operatorname{End}\left(\mathcal{H}_{0}\right) \ni \mathcal{O}_{A_{i}} \longleftrightarrow \omega_{i} \in H^{1}(\mathcal{M})
$$

such that

$$
\int_{\mathbb{R}_{+}^{m-1}} d^{m} \tau\left\langle\mathcal{O}_{\left\{A_{1}\right.} G e^{-\tau_{2} H} \mathcal{O}_{A_{2}} \ldots G e^{-\tau_{m} H} \mathcal{O}_{\left.A_{m}\right\}}\right\rangle_{B}^{A}=\int_{\mathcal{M}} \tilde{\omega}^{A} \wedge \operatorname{MP}\left(\omega_{1}, \ldots, \omega_{m} ; \omega_{B}\right),
$$

where tilde stands for Poincaré duality.

So if the conjecture is valid then we have an equality of three objects of very different nature - correlator in TQM, intersection matrix $n_{B}^{A}\left(C_{1}, \ldots, C_{m}\right)$ and the expression in the r.h.s. of the above formula.

\section{Conclusion}

We have shown the geometrical pattern of TQM - a toy model of TFT. We expressed correlators via intersection numbers on target manifold and made a conjecture that they can be expressed via integral of Massey product. Now the main problem is to prove this statement.

Quantum mechanics in the setup described tn this paper is a string theory with string length equal to zero. Generalization of this theory to topological sigma-model is a very interesting problem to be solved.

Acknowledgements. The authors are grateful to A. Gorsky, D. Krotov, D. Volin, A. Chervov, M. Libanov, A. Litvinov and especially A. Losev for fruitful discussions. This work is supported in part by grant RFBR 01-02-17227.

\section{REFERENCES}

[1] D. Birmingham, M. Blau, M. Rakowski and G. Thompson, Phys. Rept. 209 (1991) 129.

[2] L. Cohen and P. Norbury, Morse Field Theory. math.GT/0509631.

[3] K. Fukaya. Morse homotopy and its quantization. AMS/IP Studies in Advanced Math. 2, (1997) pp. $409-440$

[4] K. Fukaya. Morse homotopy, $A^{\infty}$ category and Floer homologies. Proc. of Garc Workshop in Geometry, Seoul National Univ.

[5] J. Labastida. Morse Theory Interpretation of Topological Quantum Field Theories. Commun. Math. Phys. 123, 641-658 (1989)

[6] A. Losev and I. Polyubin, JETP Lett. 82 (2005) 335.

[7] V. Lysov, JETP Lett. 76, 724 (2002) [Pisma Zh. Eksp. Teor. Fiz. 76, 855 (2002)] [arXiv:hepth/0212005]. 
[8] V. Mathai and D. Quillen. Superconnections, Thom classes and equivariant differential forms. Topology 25:85-110, (1986).

[9] E. Witten. Topological Quantum Field Theory. Comm. Math. Phys. 117, 353-386 (1988).

[10] E. Witten, Topological sigma model.

[11] E. Witten, Supersymmetry and Morse Theory. J.Diff.Geom. 17 (1982) 661.

\section{Peter Koroteev}

Institute for Theoretical and Experimental Physics 117259 Moscow, Russia,

Moscow Institute of Physics and Technology

141701 Dolgoprudny, Moscow Region, Russia,

Institute FOR NuCLEAR RESEARCH

142190 Moscow, Russia

\section{ANDREY ZAYAKIN}

Institute for Theoretical and Experimental Physics 117259 Moscow, Russia,

Moscow State University

119991 Moscow, Russia,

Bogoliubov Laboratory of Theoretical Physics 141980 Dubna, Moscow region, Russia 